\title{
Anticoagulation Activity of the Modified Poly(vinyl alcohol)
}

\author{
Heung-Jae Chun, Jae-Jin KIM, and Kea-Yong KIM* \\ Membrane Laboratory, Korea Institute of Science and Technology, \\ P.O. Box 131, Cheongryang, Seoul 130-650, Korea \\ * Department of Industrial Chemistry, Hanyang University, \\ Seoul 133-791, Korea
}

(Received October 11, 1989)

\begin{abstract}
Cross-linked blendmers of the poly(vinyl alcohol) (PVA) with substituted anion have been prepared. Possibilities of the samples for the usage as biomaterial were estimated by measuring the tensile strength and elongation under dry and wet condition, and studied by in-vitro blood tests. And the contact angle of the samples were also investigated in order to examine the surface properties. The most improved anticoagulation activity was observed in XSC-3 which is a blendmer of $60 \mathrm{wt} \%$ of the sulfonated PVA and $40 \mathrm{wt} \%$ of the carboxymethylated PVA.

KEY WORDS Sulfonated Poly(vinyl alcohol) / Carboxymethylated Poly(vinyl alcohol) / Cross-linked Blendmers / Anticoagulation Activity /
\end{abstract}

Surface properties of materials affect blood compatibility significantly. It is difficult to find synthetic polymers that have both mechanical properties and blood compatibilities as biomaterials. A lot of studies or efforts has been focused on improving blood compatibility of synthetic polymers by modifying the surface of polymers with heparin. This method has some demerits that heparin can be released easily because it is ionically bound to polymer. And it has some difficulties in processing because heparin degrades at relatively low temperature, about $120^{\circ} \mathrm{C}$.

The outstanding anticoagulation activity of heparin, a natural mucopolysaccharide, is explained mainly by the high concentration of anionic groups and their steric order and conformation along the chain. ${ }^{1-3}$ Therefore a number of semisynthetic heparinoids with the polysaccharide chain, both of natural and synthetic character, were prepared, but none of them possessed any activity worth mentioning. Some rather good anticoagulation effects were observed in poly(vinyl sulfonic) acid.
Some polyelectrolytes containing sulfonyl and carboxylic groups prepared by Beugeling et al. showed anticoagulation activity. ${ }^{4}$

This paper reports the preparation of two types of the modified poly(vinyl alcohol) containing anionic groups which appear in heparin, that is, sulfate and carboxylic groups, respectively. Then they were blended and crosslinked at various ratios and the anticoagulation activity of these cross-linked blendmers were studied.

\section{EXPERIMENTAL}

\section{Material}

Poly(vinyl alcohol) (Elvanol 71-30, M; 52,000 , du Pont) was used after drying under reduced pressure. Extra pure grade glutaraldehyde (Japan, Kokusan Co.) was used as cross-linking agent. All the other chemicals used in this work, acids and hydroxides, were extra pure grade from Kanto Chemicals Co. (Japan). 
Carboxymethylation of $P V A$

An aqueous PVA-NaOH solution containing $10 \mathrm{wt} \%$ of PVA and $6 \mathrm{wt} \%$ of $\mathrm{NaOH}$ was prepared. This solution was heated up to $80^{\circ} \mathrm{C}$ and stirred intensely for $24 \mathrm{~h}$ to introduce alkoxide groups into PVA chain. Monochloroacetic acid was added into the polymer solution ${ }^{5}$ with a concentration ranging from 2 to $12 \mathrm{wt} \%$. These solutions were stirred sufficiently for $24 \mathrm{~h}$ at $40^{\circ} \mathrm{C}$ followed by the neutralization with $\mathrm{NaOH}$ solution, washing with ethanol and acetone consecutively.

\section{Sulfonation of PVA}

We used Kokawa's method., ${ }^{6,7}$ Different amounts of sulfuric acid were added into the $10 \mathrm{wt} \%$ of aqueous PVA solution and stirred violently at $30^{\circ} \mathrm{C}$ for $24 \mathrm{~h}$. These solutions were neutralized by $\mathrm{Na}_{2} \mathrm{CO}_{3}$ followed by washing with ethanol and acetone consecutively. Concentrations of sulfuric acid used in this experiment were one to five times higher than those of $\mathrm{OH}$ groups in PVA.

In the cases of carboxymethylation and sulfonation, salts produced by the neutralization were eliminated with a semi-permeable membrane, comprising of cellulose acetate.

\section{Preparation of Cross-Linked Blendmer}

Blends of the aqueous sulfonated PVA (S-PVA) and carboxymethylated PVA (CPVA) solutions were prepared by the use of S-PVA and C-PVA whose substitution efficiencies were $25 \%$, respectively. For these cases, weight ratios of S-PVA and C-PVA vary from $1: 4$ to $4: 1$, whose total weight percent was fixed a constant, $10 \mathrm{wt} \%$. Hydrochloric acid, as an acid catalyst, was added gradually into this polymer solution until the solution reaches at $\mathrm{pH}$ 1. And it was cast onto a clear PMMA plate and gradually cross-linked as gel state at room temperature for $48 \mathrm{~h} .^{8}$ In this crosslinking procedure, glutaraldehyde solution of $2 \mathrm{wt} \%$ was used.

\section{Degree of Swelling}

The weight of completely dried samples were measured directly. And these samples were dipped into the petri dish filled with water whose temperature was maintained at $37^{\circ} \mathrm{C}$ in an incubator for two weeks. The degree of swelling, $Q_{\mathrm{w}}(\%)$, of these samples were calculated with following equation, $Q_{\mathrm{w}}(\%)=$ $\left.\left[X_{2}-X_{1}\right) / X_{1}\right] \times 100$, where $X_{1}$ is the weight of dried samples and $X_{2}$ is the weight of swollen sample.

\section{Tensile Strength and Elongation under Dry and Wet State}

The tensile strength and elongation under dry and wet condition were measured according to the method of ASTM D-638 by use of Instron (Instron Co., M10-4200) at a crosshead speed of $10 \mathrm{~mm} \mathrm{~min}^{-1}$ and $5 \mathrm{kgf}$ load cell was used.

\section{Critical Surface Tension ${ }^{9}$}

The measurement of contact angle was carried out using contact angle goniometer (Mg-1, Erma Optical Co., Japan) . A drop of organic liquid such as glycerin, formamide, diethylene glycol, oleic acid, 1,2-dichloroethane, and $n$-buthyl alcohol was placed onto polymer film, and the contact angles were measured at least 5 different sites of a sample. After determined with these measured values of contact angle, values of $\cos \theta$ of the sample were plotted according to surface tensions of fluids used in this test.

Critical surface tensions of the polymer were computed by extrapolation of plotted points up to $\cos \theta=1$ using a computing integrator (SP-4200, Spectra Physics Co.).

\section{Column Method ${ }^{10}$}

Samples were coated onto the inside wall of the PVC tubing ( $50 \mathrm{~cm}$ length, $\phi 3 \mathrm{~mm}$ ). Some $\mathrm{ml}$ of fresh human blood was collected from the healthy male donors between 24 and 27 years of age with a disposable syringe, and was passed through the tube coated with polymer 
for $3 \mathrm{~min}$ at a flow rate of $1 \mathrm{ml} \mathrm{min}{ }^{-1}$. Passed blood was mixed with anticoagulant (EDTA) in the sampling bottle to determine the numbers of platelets by platelet counter (Coulter counter, model S-plus).

\section{Lee-White Test}

Samples were coated onto the inside wall of test tube $15 \mathrm{~mm} \times 125 \mathrm{~mm}$ in size (Corning Ltd.). $2 \mathrm{ml}$ of fresh human blood was brought into the test tubes to contact with the coated polymer and the rate of clotting time was measured.

\section{Depositing Method ${ }^{11}$}

A sheet of polymer film was prepared in size of $1 \mathrm{~cm}$. This was dipped into a test tube filled with fresh human blood for $3 \mathrm{~min}$, took out of the glass tube, and washed with $0.9 \mathrm{wt} \%$ saline solution. This film was dipped into the saline solution of glutaraldehyde $(1.25 \%)$ in order to fix the adhered platelets, freeze dried and finally gold coated for the scanning electron micrographs (SEM). We used Hitachi S-510 scanning electron microscope.

\section{RESULTS AND DISCUSSION}

The carboxymethylation was confirmed by FT-IR spectroscopy. Figure 1 shows FT-IR spectra of carboxymethylated PVA. Because of the neutralization reaction, the substitution of the acidic porton in the carboxylic acid function with a different cation causes the characteristic absorptions of the carboxylic acid to vanish. The acid carbonyl absorption around $5.8 \mu \mathrm{m}$ $\left(1725 \mathrm{~cm}^{-1}\right)$ is replaced by a strong band around $6.2 \mu \mathrm{m}\left(1615 \mathrm{~cm}^{-1}\right){ }^{12}$

The substitution ratio of carboxymethylated PVA was determined by acid-base titration. Figure 2 shows that the substitution ratio is increased with increasing the concentration of monochloroacetic acid.

Contents of elements which consist of the sulfonated PVA were examined in order to determine the substitution ratio by sulfur

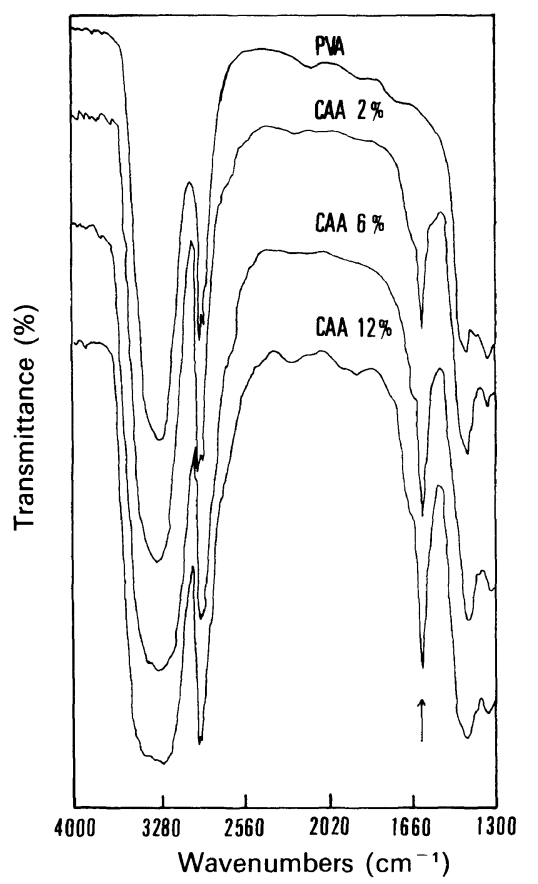

Figure 1. IR spectra of carboxymethylated PVA.

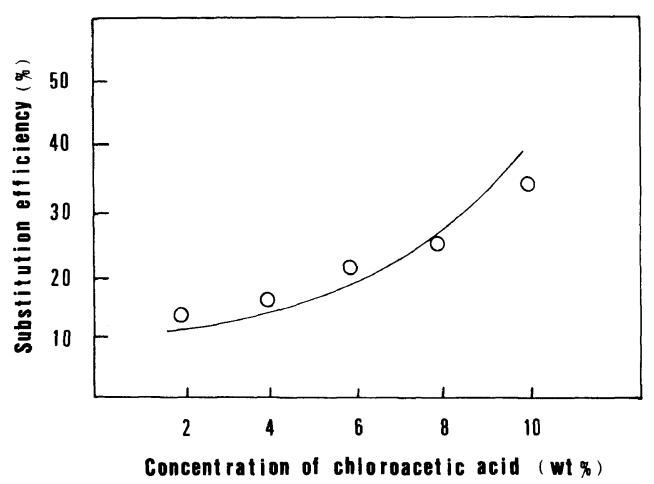

Figure 2. Substitution ratio of carboxymethylated PVA.

analysis and elementary analysis (E. A. Perkin-Elmer 240 DS).

The degradation of PVA accompanied by the sulfonation was confirmed by solution viscometry. Figure 3 shows the relation between the sulfonation and the degradation ratio of PVA. Though the substitution ratio is increased with increasing the concentration of sulfuric acid, the degradation is also increased. 


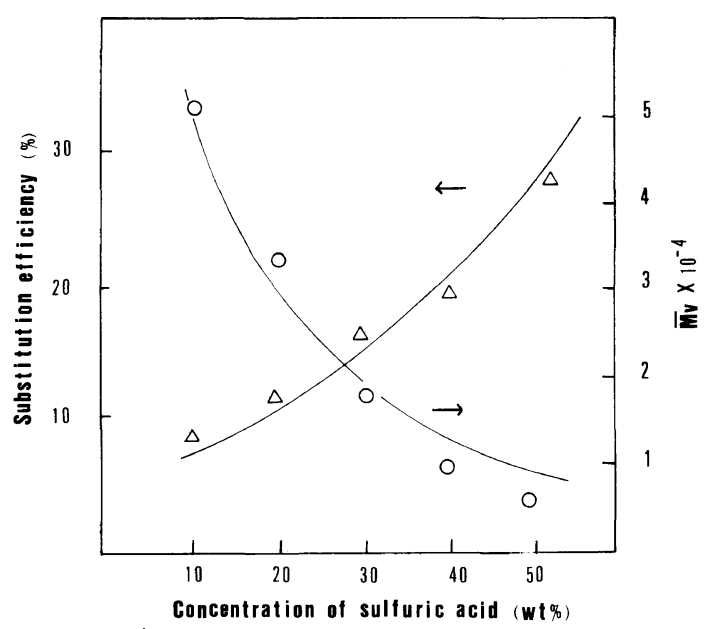

Figure 3. Relation between sulfonation and degradation ratio.

Table I. Degree of swelling

\begin{tabular}{lc}
\hline Sample & Water content $/ \%$ \\
\hline X-PVA & 420 \\
XS-PVA & 382 \\
XC-PVA & 297 \\
XSC-1 & 314 \\
XSC-2 & 326 \\
XSC-3 & 331 \\
XSC-4 & 344 \\
\hline
\end{tabular}

X-PVA : cross-linked PVA

XS-PVA : cross-linked sulfonated PVA

XC-PVA : cross-linked carboxymethylated PVA

XSC-1 : cross-linked blendmer (S : $\mathrm{C}=1: 4)$

XSC-2 : cross-linked blendmer $(\mathrm{S}: \mathrm{C}=2: 3)$

XSC-3 : cross-linked blendmer $(\mathrm{S}: \mathrm{C}=3: 2)$

XSC-4 : cross-linked blendmer $(\mathrm{S}: \mathrm{C}=4: 1)$
Figure 4 shows IR spectra of cross-linked blendmer. This presents an absorption band at $1140 \mathrm{~cm}^{-1}$, characteristics of the crystalline region of PVA. And this band is decreased with increasing the concentration of cross-linking agent. On the contrary, the carbonyl absorption of acetal group around $1100 \mathrm{~cm}^{-1}$ is increased gradually.

The degree of swelling of samples are shown in Table I. In the case of cross-linked blendmer, the degree of swelling is increased with increasing the concentration of sulfonated

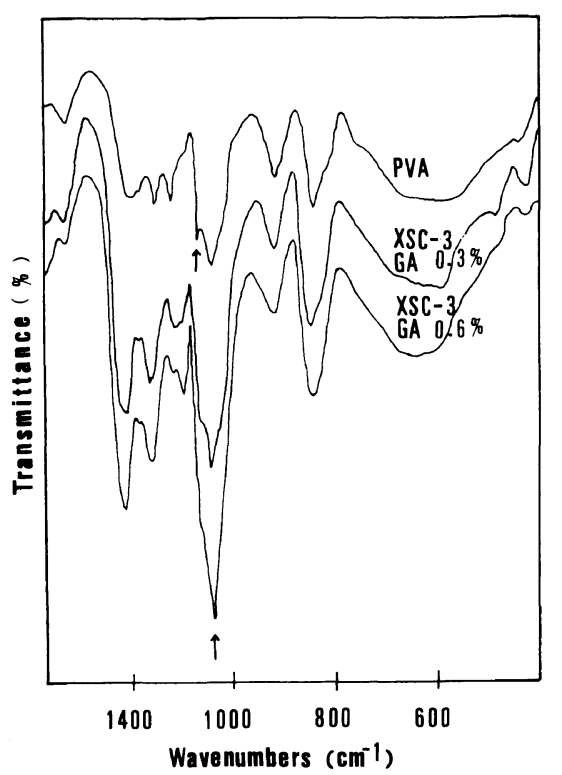

Figure 4. IR spectra of crosslinked blendmer.

Table II. Tensile strength and elongation under dry and wet state

\begin{tabular}{|c|c|c|c|c|c|}
\hline \multicolumn{3}{|l|}{ Dry state } & \multicolumn{3}{|c|}{ Swollen state } \\
\hline \multirow{2}{*}{ Sample } & Tensile strength & Elongation & \multirow{2}{*}{ Sample } & Tensile strength & Elongation \\
\hline & $\mathrm{kg} \mathrm{mm}^{-2}$ & $\%$ & & $\mathrm{~kg} \mathrm{~mm}^{-2}$ & $\%$ \\
\hline PVA & 7.30 & & & & \\
\hline X-PVA & 12.427 & 27 & X-PVA & 2.38 & 82 \\
\hline XS-PVA & 0.730 & 12 & XS-PVA & 0.33 & 46 \\
\hline XC-PVA & 3.762 & 180 & XC-PVA & 1.14 & 379 \\
\hline XSC-1 & 4.482 & 160 & XSC-1 & 0.70 & 290 \\
\hline XSC-2 & 3.980 & 146 & $\mathrm{XSC}-2$ & 0.66 & 270 \\
\hline $\mathrm{XSC}-3$ & 2.714 & 126 & $\mathrm{XSC}-3$ & 0.61 & 207 \\
\hline XSC-4 & 1.547 & 122 & XSC-4 & 0.46 & 173 \\
\hline
\end{tabular}


PVA, in the order of XSC-1, XSC-2, XSC-3, and XSC-4.

The tensile strength and elongation under dry and wet condition of these samples are

Table III. Critical surface tension of the samples

\begin{tabular}{lc} 
& Critical surface tension \\
\cline { 2 - 2 } Sample & dyn cm $^{-1}$ \\
\hline PVA & 37.80 \\
X-PVA & 29.51 \\
XS-PVA & 28.94 \\
XC-PVA & 24.16 \\
XSC-1 & 25.07 \\
XSC-2 & 25.23 \\
XSC-3 & 25.60 \\
XSC-4 & 26.14 \\
\hline
\end{tabular}

Table IV. Blood clotting time of samples

\begin{tabular}{lcc}
\hline Sample & Clotting time/second & Clotting ratio \\
\hline X-PVA & 408 & 1.29 \\
XC-PVA & 446 & 1.41 \\
XS-PVA & 452 & 1.43 \\
XSC-1 & 460 & 1.46 \\
XSC-2 & 499 & 1.58 \\
XSC-3 & 638 & 2.03 \\
XSC-4 & 627 & 1.99 \\
Glass & 315 & 1.00 \\
\hline
\end{tabular}

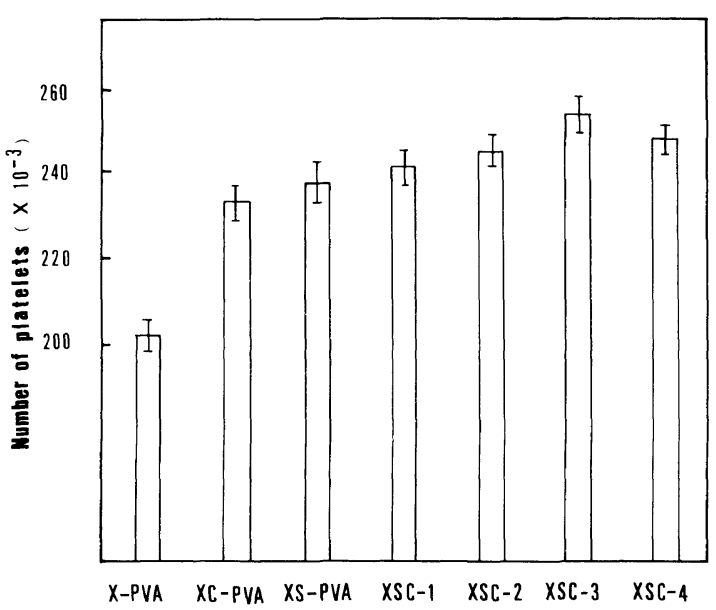

Figure 5. The number of platelets passed through polymer coated column. shown in Table II. The tensile strength and elongation of the cross-linked blendmers are decreased with increasing the concentration of sulfonated PVA. This effect can be explicable because the sulfonated PVA has brittle mechanical property with its decreasing molec-
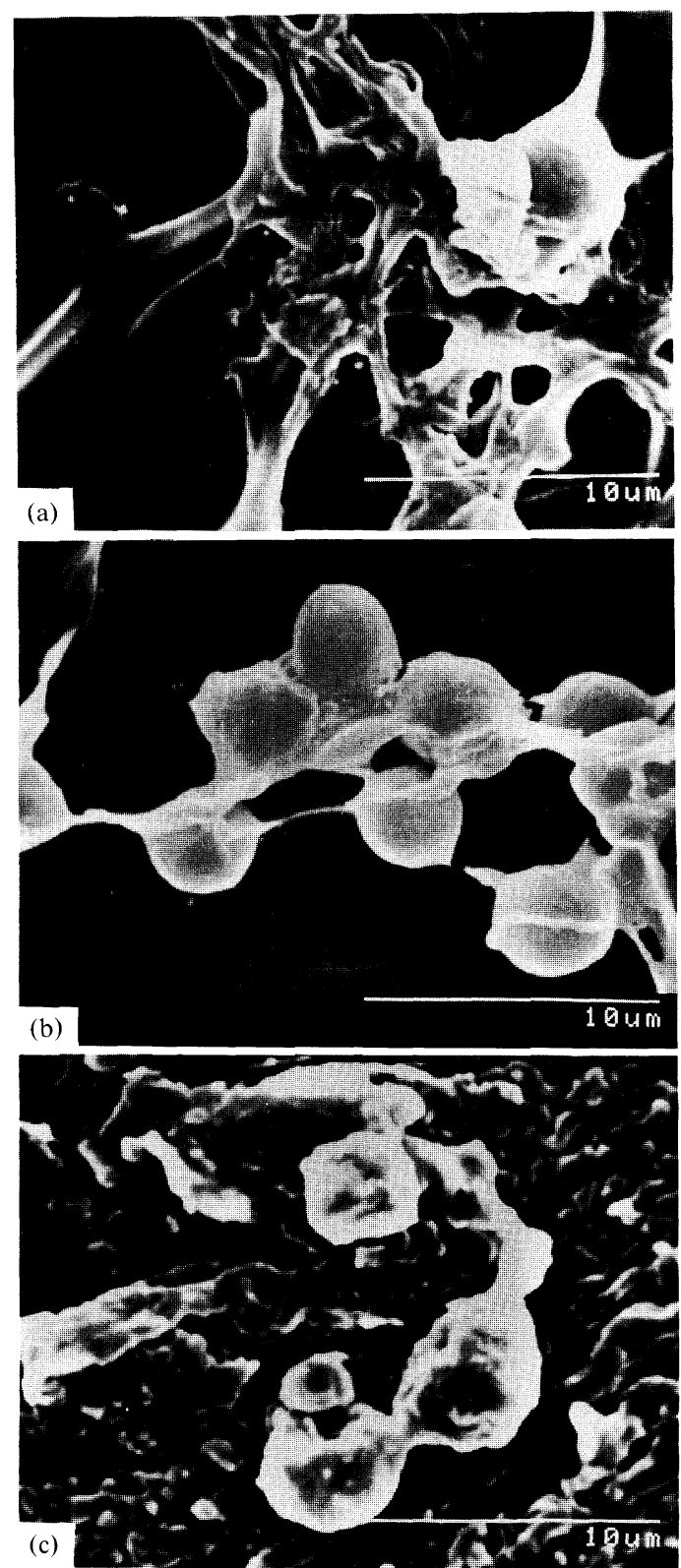

Figure 6. Scanning electron micrographs of adhered platelets onto (a) glass, (b) PVC and (c) X-PVA's surface. 


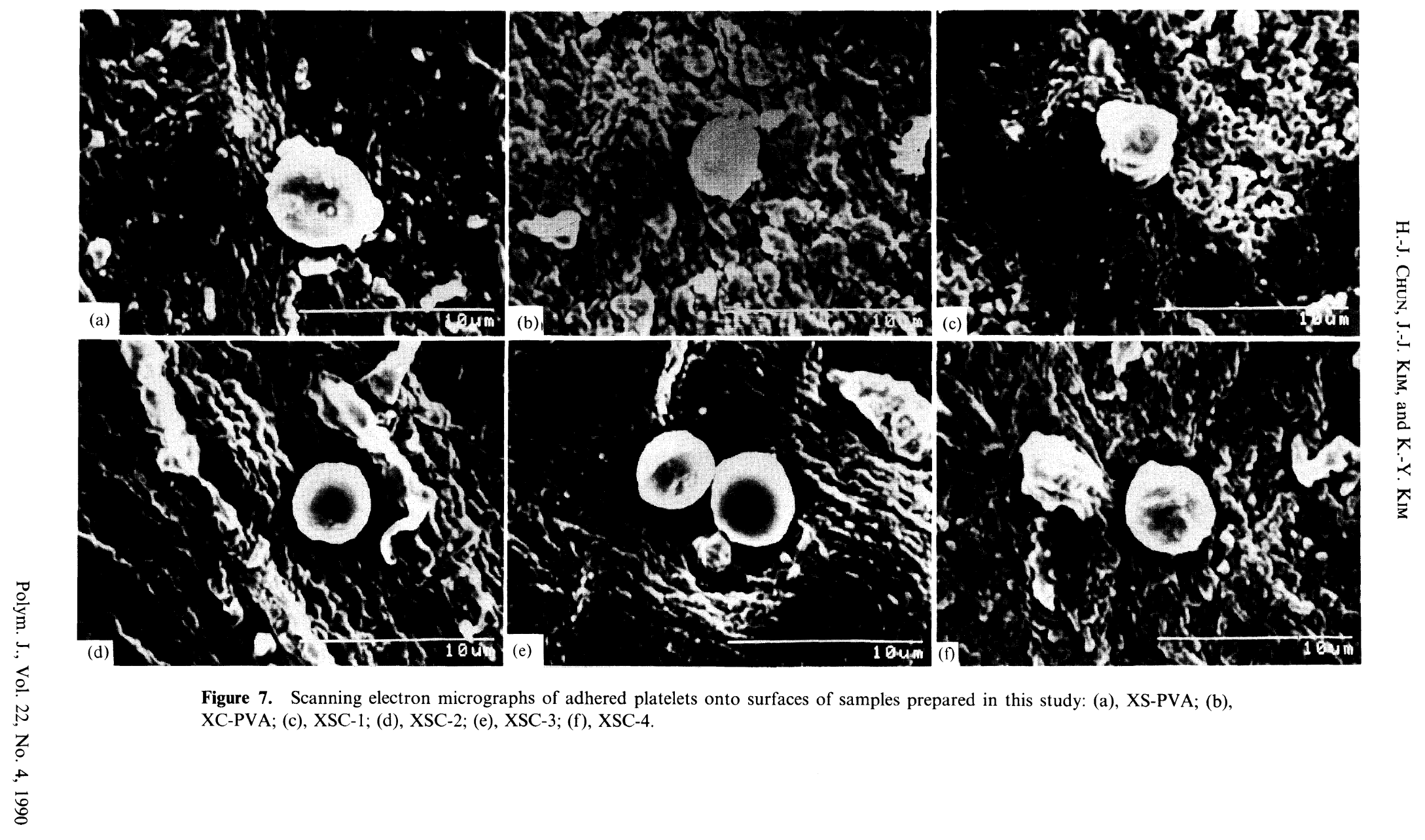


ular weight by the degradation.

As compared with dry condition, tensile strength of all swollen samples show a trend of decrease, but have more improved values of elongation than those of dried samples.

Table III shows critical surface tensions of prepared samples. Critical surface tensions of cross-linked samples are in the range of $20-30$ dyn $\mathrm{cm}^{-1}$.

In Table IV experimental results of in-vitro blood clotting test on prepared samples are shown. And numbers of platelets passed through polymer coated column determined by Coulter counter are shown in Figure 5.

From these results, cross-linked blendmers have more predominant anti-coagulant properties than XC-PVA and XS-PVA itself. Among them, the cross-linked blendmer XSC-3 which contains $60 \mathrm{wt} \%$ of the sulfonated PVA is the best.

Scanning electron micrographs of blood platelets attached onto the surface of tested materials are shown in Figure 6. In the case of glass, it is no longer possible to distinguish each original shape of adhered platelets.

In the case of the cross-linked PVA membrane, platelets are deformed gradually and formed pseudopodia. On the surface of the PVC tubing used in column test, the deformation and aggregation of adhered platelets are so significant compared with those on the other materials' surface.

Figure 7 shows blood platelets attached onto the surface of modified PVA prepared in this study. These platelets are deformed scarcely and have no pseudopodia and fibrin net.

Though some authors ${ }^{13,14}$ believe that the anticoagulation activity of heparin is influenced mostly by the $\mathrm{N}$-sulfonated group, according to some reports, ${ }^{15-17}$ sulfamate groups do not affect the anticoagulation activity of heparin. But it is believed that the predominant anticoagulation activity of heparin is due to the effect of anionic groups; sulfate and carbonyl groups. ${ }^{18}$ It was proved that when $-\mathrm{OSO}_{3} \mathrm{H}$ and $-\mathrm{COOH}$ groups were blocked the activity of heparin was disappeared. And activity remains preserved only with coexisting state of sulfate and carboxyl groups. ${ }^{19}$

In this study, that the modified PVA, sulfonated and carboxymethylated PVA, shows more improved anticoagulation activity than the pure PVA is recognized due to the effect of polar groups introduced into the PVA chain.

Cross-linked blendmers which consist of the sulfonated PVA and carboxymethylated PVA show more improved blood compatibility than the XS-PVA and XC-PVA itself. And the anticoagulation activity of modified PVA is also improved by the transformed surface property; critical surface tensions of samples are about $25 \mathrm{dyn}^{-1} \mathrm{~cm}^{20}$

\section{CONCLUSIONS}

It is known that O-sulfate and carboxylic groups have important influence on the anticoagulation. And they have more significant effect when two groups are coexisted. Results examined in this study are satisfied with these reported theory; cross-linked belndmers show more improved anticoagulation activity than the XS-PVA and XC-PVA itself. And critical surface tensions of cross-linked blendmers are in the range of $20-30 \mathrm{dyn} \mathrm{cm}^{-1}$ satisfying Baier's or Lymann's assertion.

\section{REFERENCES}

1. P. Bernfeld, "The Amino Sugars," Vol. IIB, Academic Press, New York, 1966, p 125.

2. F. A. Pitlick and R. L. Lundbald, J. Biomed. Mater. Res., 3, 95 (1969).

3. H. P. Gregor, Biomed. Appl. Polym., 7, 51 (1975).

4. T. Beugeling and L. Van Der Does, J. Biomed. Mater. Res., 8, 375 (1974).

5. M. Hida, Kogyo Kakaku Zasshi, 55, 221 (1952).

6. K. Kokawa and S. Nozakura, Kobunshi Kakaku, 24, 801 (1967).

7. K. Kokawa and S. Nozakura, Kobunshi Kakaku, 25, 19 (1968).

8. K. Y. Kim, D. S. Min, and H. J. Chun, Polymer (Korea), 11, 246 (1987).

9. H. Y. Fox and W. A. Zisman, J. Colloid. Sci., 7, 109 
(1952).

10. K. Kataoka, Macromol. Chem., 179, 1121 (1978).

11. A. W. Neuman and M. A. Moscarello, J. Polym. Sci., Polym. Symp., 66, 391 (1979).

12. "The Aldrich Library of Infrared Spectra," 3rd ed., 1981, pp 283-321.

13. Z. Yosizawa, T. Kotoku, F. Yamauchi, and M. Matsuno, Biochim. Biophys. Acta, 141, 358 (1967).

14. S. E. Lasker and S. S. Stivala, Arch. Biochem. Biophys., 115, 360 (1966).

15. J. A. Cifonelli and J. King, Biochim. Biophys. Acta,
320, 331 (1973).

16. L. Van Der Doew, T. Beugeling, P. E. Froehling, and A. Banties, J. Polym. Sci., Polym. Symp., 66, 337 (1979).

17. M. Sorm, S. Nespurek, K. Mrkvickova, and J. Kalal, J. Polym. Sci., Polym. Symp., 66, 349 (1979).

18. R. L. Taylor, J. E. Snively, H. E. Conrad, and J. A. Cifonelli, Biochemistry, 12, 3633 (1973).

19. A. B. Foster and A. J. Juggard, Adv. Carbohyd. Chem., 10, 335 (1955).

20. R. E. Baier, Federation Proceedings, 30, 1526 1971). 